\title{
CXCR4 promotes cisplatin-resistance of non-small cell lung cancer in a CYP1B1-dependent manner
}

\author{
SONGPING XIE ${ }^{*}$, ZHENBO TU $^{2 *}$, JIE XIONG $^{2 *}$, GANJUN KANG $^{1}$, LINA ZHAO $^{3}$, WEIDONG HU $^{4}$, HAIYAN TAN $^{5}$, \\ KINGSLEY MIYANDA TEMBO ${ }^{2}$, QIANSHAN DING ${ }^{6}$, XINZHOU DENG $^{2}$, JIE HUANG $^{1}$ and QIUPING ZHANG ${ }^{2}$ \\ ${ }^{1}$ Department of Thoracic Surgery, Renmin Hospital of Wuhan University, Wuhan, Hubei 430060; \\ ${ }^{2}$ Department of Immunology, School of Basic Medical Science, Wuhan University, Wuhan, Hubei 430071; \\ ${ }^{3}$ Department of Pathology, Renmin Hospital of Wuhan University, Wuhan, Hubei 430060; \\ ${ }^{4}$ Department of Thoracic Oncology, Zhongnan Hospital, Wuhan University, Wuhan, Hubei 430071; \\ Departments of ${ }^{5}$ Gastrointestinal Surgery, and ${ }^{6}$ Gastroenterology, \\ Renmin Hospital of Wuhan University, Wuhan, Hubei 430060, P.R. China
}

Received June 5, 2016; Accepted October 31, 2016

DOI: $10.3892 /$ or.2016.5289

\begin{abstract}
Chemoresistance is the main cause of treatment failure and high mortality in advanced lung cancer. Cisplatin, an important chemotherapeutic agent for lung cancer, has been observed to show enormously reduced chemotherapeutic efficacy owing to the development of chemoresistance. CXCR4, a stromal-derived-factor-1 specific chemokine receptor, is highly expressed in non-small cell lung cancer (NSCLC) tissues and participates in cancer progression by regulating cell growth, apoptosis or invasion. In this study, we therefore investigated whether CXCR4 plays a role in the cisplatin associated resistance in NSCLC. We detected the expression of CXCR4 in tissue specimens from 64 NSCLC patients by immunohistochemistry. Cisplatin-resistant NSCLC cells A549/DDP and its parental A549 cells were employed in this study. RNA interference was performed to silence the CXCR4. The influence of CXCR4 on tumor cell chemoresistance, apoptosis and growth, as well as the relationship between CXCR4 and the expression of cytochrome p450 associated molecule CYP1B1 in NSCLC were evaluated. Finally, we found CXCR4 was
\end{abstract}

Correspondence to: Professor Qiuping Zhang, Department of Immunology, School of Basic Medical Sciences, Wuhan University, 185 Donghu Road, Wuchang, Wuhan, Hubei 430071, P.R. China E-mail: qpzhang@whu.edu.cn

Professor Jie Huang, Department of Thoracic Surgery, Renmin Hospital of Wuhan University, 238 Jiefang Road, Wuchang, Wuhan, Hubei 30060, P.R. China

E-mail: doctor_huangjie@126.com

*Contributed equally

Abbreviations: NSCLC, non-small cell lung cancer; CYP1B1, cytochrome P450 1B1

Key words: non-small cell lung cancer, chemoresistance, CXCR4, CYP1B1 significantly highly expressed in cisplatin-resistant NSCLC patients and the A549/DDP cell line. CXCR4 inhibition by siRNA reversed chemoresistance and decreased tumor cell proliferation. Bioinformatics analysis showed that the expression of CYP1B1 had a positive correlation with CXCR4, the CYP1B1 silencing significantly decreased CXCR4 expression levels and cisplatin resistance. Immunohistochemistry also verified that CYP1B1 was upregulated in NSCLC tissues of cisplatin-resistant patients. In conclusion, our results indicate that overexpression of CXCR4 in NSCLC promotes cisplatin resistance via CXCR4-mediated CYP1B1 upregulation. Thus, it can be used as a potential therapeutic target in NSCLC chemoresistance patients and be used as a clinical predictor of cisplatin response.

\section{Introduction}

Lung cancer is one of the leading causes of cancer-related deaths worldwide in both males and females, with non-small cell lung cancer (NSCLC) accounting for $\sim 87 \%$ of all lung cancer cases (1). At diagnosis, most patients present with advanced NSCLC stages, mainly due to the lack of effective early diagnosis techniques. Presently, chemotherapy is a major treatment to prolong disease-free survival for these patients. However, little success has been made in improving patient survival rates, as the 5-year survival rates have remained at $\sim 18.2 \%(2,3)$. Researchers have reported the occurrence of intrinsic or acquired resistance, as the main challenge associated with chemotherapeutic resistance in cancer cells (4). Cisplatin-based chemotherapy is frequently used in the treatment of NSCLC. The resistance obtained following continuous therapy limits the benefit of cisplatin in cancer treatment, but the exact mechanism of cisplatin resistance in lung cancer remains unknown.

CXCR4, a seven-transmembrane G protein-coupled receptor, is a physiological receptor for stromal-derivedfactor-1 (5) which is highly expressed in various types of human tumors, including lung cancer $(6,7)$, esophageal 
cancer (8), gastric cancer (9), endometrial cancer (10) and breast cancer (11). A number of studies have demonstrated the vital role of CXCR4 in cancer cell survival, proliferation, invasion and metastasis, thus high expression of CXCR4 has been shown to be associated with a poor prognosis in cancer patients. Nakamura et al reported that CXCR4 signaling is crucial for drug resistance in bone marrow-disseminated tumor cells (12). While Li et al observed that the overexpression of CXCR4 was significantly associated with cisplatin-based chemotherapy resistance in epithelial ovarian cancer (13). Although this suggests a possible role for CXCR4 in development of chemotherapy resistance, the precise role of CXCR4 in cisplatin resistance of lung cancer remains largely unexplored.

In this study, we detected the expression of CXCR4 in NSCLC patients and cell lines, and demonstrated the possible effect of CXCR4 knockdown on cisplatin-induced apoptosis. In addition, we also explored the possible molecular mechanisms by which CXCR4 possibly regulates cisplatin resistance in NSCLC.

\section{Materials and methods}

NSCLC tissue samples. A total of 64 lung cancer specimens were collected from patients with primary NSCLC surgical resection at Renmin Hospital of Wuhan University from May 2012 to April 2015. None of the subjects had received any chemotherapy and radiotherapy prior to surgery. All of these patients were treated with cisplatin-based chemotherapy after surgery according to the NCCN guideline every 3 weeks for four cycles. Patients were followed-up by telephone until recurrence/metastasis or until May 2016. All patients were monitored by chest computed tomography scan, abdominal ultrasonography and brain magnetic resonance imaging every 3 months in the first year after surgery, or immediately when a recurrence/metastasis was suspected. A whole-body fludeoxyglucose positron emission tomography/CT was performed when needed. The WHO grades and clinical features of these patients were summarized in Table I. Patients with disease progression or recurrence 12 months after surgery were defined as being chemoresistance, whereas those without recurrence or recurrence $>12$ months after surgery were defined as chemosensitive. There were 32 NSCLC patients who are sensitive to cisplatin treatment and 32 NSCLC patients with cisplatin chemoresistance. This study was carried out in accordance with the principles of the Helsinki Declaration and approved by the ethics committee of the Renmin Hospital of Wuhan University.

NSCLC cell lines. A549 was obtained from American Type Culture Collection, cisplatin resistant A549 cell line (A549/DDP) was obtained from Fudan Institutes of Biomedical Sciences Cell Center, and the cells were maintained in Dulbecco's modified Eagle's medium with high glucose (Gibco, USA) supplemented with $10 \%$ fetal bovine serum (FBS; Hyclone, USA), containing $100 \mathrm{U} / \mathrm{ml}$ penicillin and $100 \mathrm{mg} / \mathrm{ml}$ streptomycin. Both cell lines were cultured in a $5 \%$ $\mathrm{CO}_{2}$ air incubator at $37^{\circ} \mathrm{C}$ and passaged using $0.25 \%$ trypsinEDTA (Gibco) when they reached confluence. Cisplatin was purchased from Sigma Chemical Co. (USA).
Table I. Comparison of clinical characteristics between patients with low and high CXCR4 cell content.

\begin{tabular}{|c|c|c|c|c|}
\hline Clinical factor & $\begin{array}{c}\text { Total } \\
\text { patients } \\
(\mathrm{n}=64)\end{array}$ & $\begin{array}{c}\text { High } \\
\text { expression } \\
(n=46)\end{array}$ & $\begin{array}{c}\text { Low } \\
\text { expression } \\
(n=18)\end{array}$ & $\mathrm{P}$-value \\
\hline \multicolumn{5}{|l|}{ Age (years) } \\
\hline$\leq 65$ & 15 & 12 & 3 & 0.424 \\
\hline$>65$ & 49 & 34 & 15 & \\
\hline \multicolumn{5}{|l|}{ Gender } \\
\hline Male & 44 & 35 & 9 & 0.043 \\
\hline Female & 20 & 11 & 9 & \\
\hline \multicolumn{5}{|l|}{ T stage } \\
\hline $\mathrm{T} 2$ & 43 & 26 & 17 & $0.004^{\mathrm{b}}$ \\
\hline $\mathrm{T} 3$ & 14 & 13 & 1 & \\
\hline $\mathrm{T} 4$ & 7 & 7 & 0 & \\
\hline \multicolumn{5}{|l|}{$\mathrm{N}$ stage } \\
\hline No & 36 & 24 & 12 & $0.490^{\mathrm{b}}$ \\
\hline N1 & 7 & 6 & 1 & \\
\hline $\mathrm{N} 2$ & 21 & 16 & 5 & \\
\hline \multicolumn{5}{|l|}{ M stage } \\
\hline M0 & 62 & 46 & 16 & $0.076^{\mathrm{a}}$ \\
\hline M1 & 2 & 0 & 2 & \\
\hline \multicolumn{5}{|l|}{ Histology } \\
\hline Adenocarcinoma & 39 & 26 & 13 & 0.247 \\
\hline $\begin{array}{l}\text { Squamous } \\
\text { carcinoma }\end{array}$ & 25 & 20 & 5 & \\
\hline \multicolumn{5}{|l|}{ Drug-resistance } \\
\hline Yes & 32 & 27 & 5 & 0.026 \\
\hline No & 32 & 19 & 13 & \\
\hline
\end{tabular}

${ }^{\mathrm{a}}$ Fisher's exact test. ${ }^{\mathrm{b}}$ Likelihood ratio.

Immunohistochemistry. Paraffin-embedded tissue sections were dewaxed and rehydrated, and antigen retrieval was performed by microwaving in $10 \mathrm{mM}$ sodium citrate buffer, $\mathrm{pH} 6.0$, for $20 \mathrm{~min}$. Sections were then incubated with $3 \%$ hydrogen peroxide for $30 \mathrm{~min}$ at room temperature to block endogenous peroxidase, then blocked in $10 \%$ normal goat serum for $0.5 \mathrm{~h}$. Immunostaining was performed by incubating with anti-CXCR4 (1:500, ab124824, Abcam Corp., UK) or anti-CYP1B1 (1:1,000, ab32649, Abcam Corp.) at $4^{\circ} \mathrm{C}$ overnight. Slides were then washed in PBS and incubated with secondary antibody (anti-rabbit detection system; Boster, China) for $30 \mathrm{~min}$ at $37^{\circ} \mathrm{C}$. Staining was visualized with 3,3-diaminobenzidine and counterstained with hematoxylin.

Quantitative $R T-P C R$. Total RNA was extracted from A549 and A549/DDP cells using TRIzol (Invitrogen, USA) according to the manufacturer's instructions and quantified by Nanodrop 2000 (Thermo Scientific, USA). RNA $(1 \mu \mathrm{g})$ was reverse-transcribed to cDNA with random primers using 
the Promega reverse transcriptase kit (USA) according to the manufacturer's protocol. To assess gene expression, cDNAs were amplified with the SYBR ${ }^{\circledR}$ Premix Ex Taq $^{\text {TM }}$ II (Tli RNaseH Plus) (Takara, Japan) using the QuantStudio 6 Flex Real-Time PCR system (Life Technologies, USA). The relative level was calculated by the comparative $\mathrm{Ct}$ method $\left(2^{-\Delta \Delta \mathrm{Ct}}\right)$. The sequences of specific primers were as follows: CXCR4, forward, 5'-CCGTGGCAAACTGGTACTTT-3'; reverse, 5'-GACGCCAACATAGACCACCT-3'. CYP1B1, forward, 5'-AAGTTCTTGAGGCACTGCGAA-3'; reverse, 5'-GGCC GGTACGTTCTCCAAAT-3'.

Western blot analysis. Cells were washed with ice cold PBS and lysed by RIPA supplemented with protease inhibitor PMSF on ice. The extracted proteins were separated by $12 \%$ SDS-polyacrylamide gel electrophoresis and transferred to PVDF membranes (Millipore, USA). The membrane was blocked with $5 \%$ non-fat milk TBS-T (0.1\% Tween-20, $100 \mathrm{nM}$ Tris- $\mathrm{HCl}, 0.9 \% \mathrm{NaCl})$ and incubated with anti-CXCR4 (1:200, sc-6190, Santa Cruz, USA) or anti-CYP1B1 $(1: 1,000$, ab32649, Abcam Corp.) with gentle shaking at $4^{\circ} \mathrm{C}$ overnight. After washing three times, the membranes were incubated with HRP-conjugated secondary antibodies for $2 \mathrm{~h}$ at room temperature. The protein bands were visualized with ECL plus western blotting detection reagents (Thermo Scientific).

Immunofluorescence assay. Cells were fixed and permeabilized with $2 \%$ paraformaldehyde and $0.5 \%$ Triton X-100. After overnight incubation with anti-CXCR4 (1:100, sc-6190, Santa Cruz), the specimens were rinsed thoroughly and treated with anti-goat antibodies, respectively, conjugated with Alexa Fluor 488 and Alexa Fluor 594 (Invitrogen), diluted $1: 400\left(1 \mathrm{~h}, 37^{\circ} \mathrm{C}\right)$. Nuclei were stained using $0.3 \mu \mathrm{M}$ DAPI (Molecular BioProbes, USA). The digital images were then captured with a cooled CCD camera and processed with the help of Photoshop (Adobe) software.

siRNA transfect assay. Cells were plated $24 \mathrm{~h}$ before transfection. At 30-50\% confluence, cells were transfected using Lipofectamine $^{\mathrm{TM}} 2000$ (Invitrogen) with siRNA duplexes specific for human CYP1B1 (RiboBio, China) or control negative control (NC) siRNA. The following were the sequences of siRNAs used in the study, CXCR4 siRNA: sense, GUGGCA AACUGGUACUUUG; antisense, CAAAGUACCAGUUUG CCAC. CYP1B1siRNA: sense, GCAUGAUGCGCAACUU CUU; antisense, AAGAAGUUGCGCAUCAUGC. In addition, mock control was included where cells were treated with Lipofectamine 2000 alone. The siRNA experiment was carried out for $48 \mathrm{~h}$ to analyse the RNA expression level by qRT-PCR and $72 \mathrm{~h}$ to analyse the protein expression level by western blot analysis.

Apoptosis assay. Cells were dual stained with the Annexin V-FITC apoptosis detection kit (BD Biosciences, USA) according to the manufacturer's protocol. Stained cells were immediately analyzed by flow cytometry (FACSAriaIII, BD Biosciences).

Cell proliferation assay. A549 and A549/DDP cells were seeded in 96 -well plate at the density of $1.0 \times 10^{4}$ cells per well overnight for adherence. The next day cells were transfected with CXCR4-siRNA or CYP1B1-siRNA. After $48 \mathrm{~h}$ of transfection, $10 \mu \mathrm{lCCK}-8$ solutions was added to each well at indicated times and incubated for another $2 \mathrm{~h}$. The absorbance of each well was obtained from Perkin-Elmer 2030 Victor $\mathrm{x}$ multilabel plate reader (Perkin-Elmer, Waltham, MA, USA) at $450 \mathrm{~nm}$.

Gene expression omnibus dataset correlation analysis. We downloaded GSE30219 (14) and GSE41271 $(15,16)$ which were the largest NSCLC datasets from Gene expression omnibus (http://www.ncbi.nlm.nih.gov/geo/) to verify the correlation of CXCR4 and CYP1B1. GSE30219 included 272 NSCLC patients and GSE41271 included 263 NSCLC patients.

Statistical analysis. Each assay was performed in triplicate and repeated a minimum of three times. Statistical analysis was performed using SPSS 19.0 or GraphPad Prism 5 software. Data are reported as means \pm SD. Statistical differences were analyzed by Student's t-test for data between control and treated groups, or a one-way analysis of variance for data from multiple groups, with the level of significance set at $\mathrm{P}<0.05$.

\section{Results}

CXCR4 expression closely correlated with NSCLC cisplatin resistance. To investigate the association between CXCR4 and cisplatin resistance in NSCLC, we first detected the expression levels of CXCR4 in 64 NSCLC patients by immunohistochemistry. We found that the levels of CXCR4 were significantly increased in cancer samples from cisplatin-resistant patients, compared to that in samples from cisplatin-sensitive patients. We categorized the NSCLC-sensitive group into negative (40.6\%), weak $(28.1 \%)$, moderate $(18.8 \%)$ and strong $(12.5 \%)$ and resistance group into negative $(15.6 \%)$, weak $(34.4 \%)$, moderate $(21.9 \%)$ and strong $(28.1 \%)$, respectively (Fig. 1A and $\mathrm{B})$. The staining scores being $1.63 \pm 1.07$ in the cisplatinresistant group and $1.03 \pm 1.06$ in the sensitive group (Fig. 1C). Furthermore, we investigated the expression levels of CXCR4 in A549 and A549/DDP cells by qPCR, western blot analysis and immunofluorescence. As shown in Fig. 1D and E, the expression levels of CXCR4 mRNA and protein in A549/DDP were significantly higher than that in A549 cells. These results indicate that CXCR4 may be involved in NSCLC cancer cisplatin resistance.

Clinical characteristics associated with CXCR4 expression in NSCLC patients. We carried out a comparative analysis of clinicopathological characteristics and associated CXCR4 expression. As shown in Table I, 75\% $(\mathrm{n}=49)$ of the patients were $>65$ years old, with $\sim 68 \%(n=44, P=0.043)$ being male patients, who accounted for $\sim 76 \%(n=35)$ of all patients with high CXCR4 expression. CXCR4 high expression was observed to be mostly associated with T2N0M0 cancer stages, indicating an increased tumor size at $\mathrm{T} 2$ stage $(\mathrm{P}=0.004)$, with no nearby lymph node invasion and no metastatic spread to other regions. CXCR4 high expression was also associated with a slightly higher percentage prevalence in adenocarcinoma as compared to squamous carcinoma and a higher drug resistance levels $(\mathrm{P}=0.026)$. This therefore suggests that high 
A

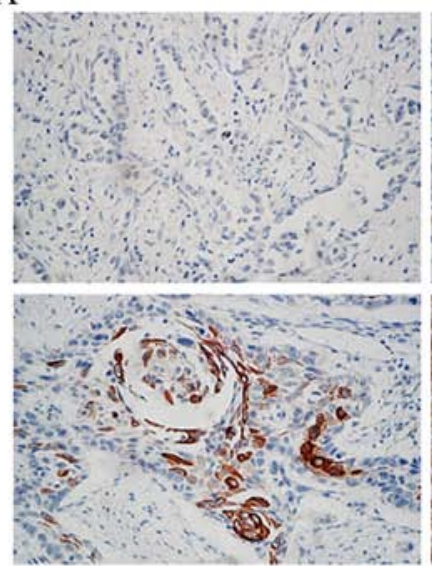

B

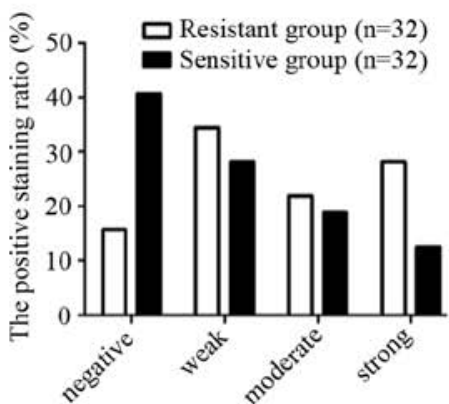

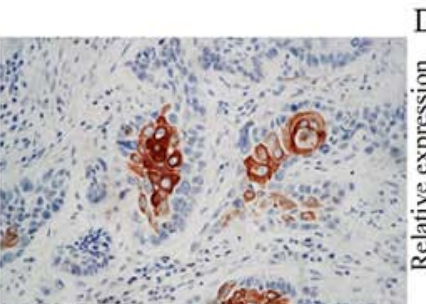

$\mathrm{D}$
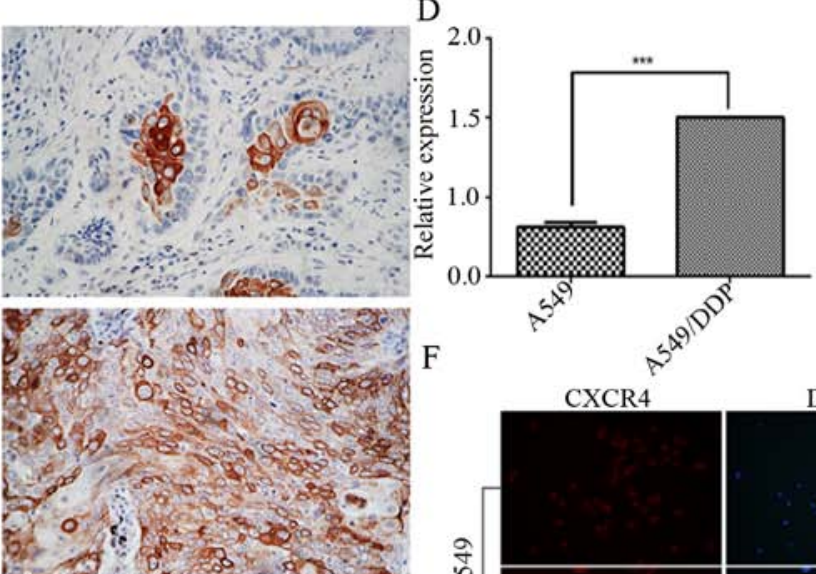

$\mathrm{C}$

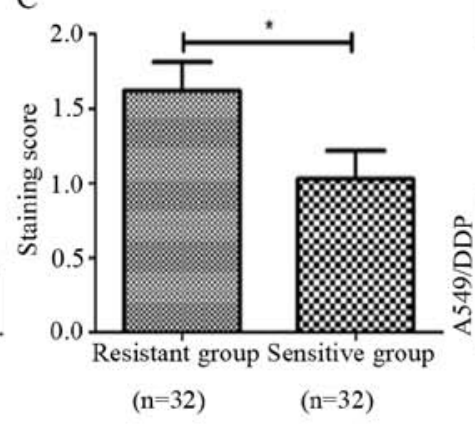

E
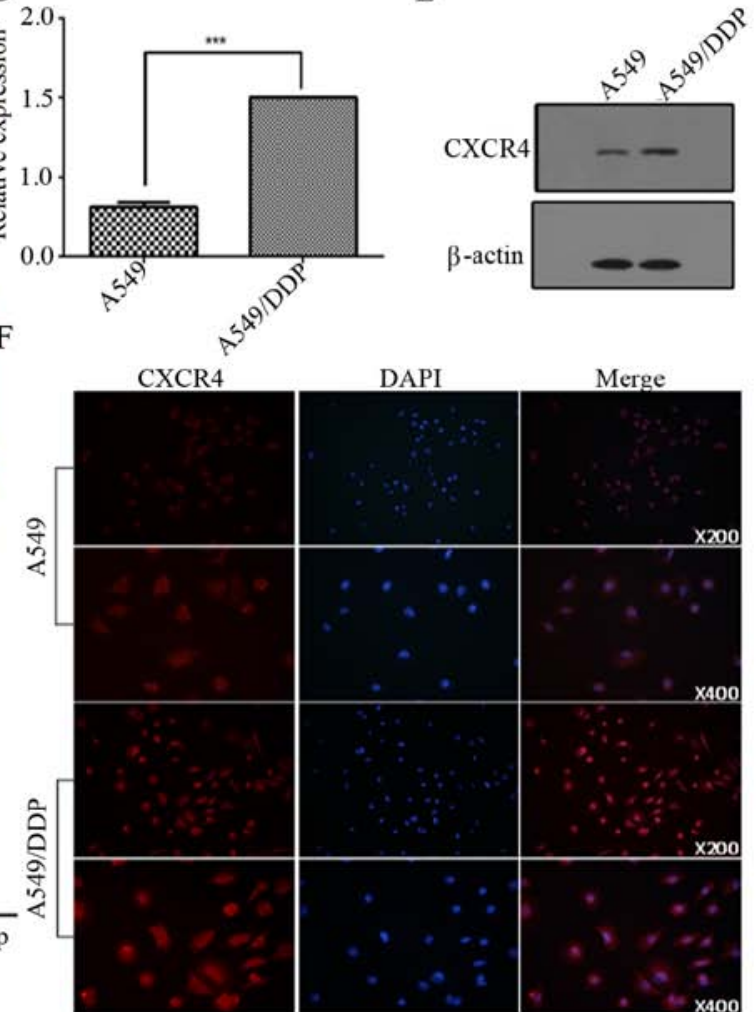

Figure 1. CXCR4 expression correlates with NSCLC cisplatin resistance. (A) Representative images of NSCLC patients by immunohistochemistry (magnification, $x 40$ ). (B) Positive staining ratio of cisplatin-resistant and sensitive NSCLC group. (C) Immunohistochemistry staining score of cisplatin-resistant and sensitive group. (D) qPCR analysis of CXCR4 expression in A549 and A549/DDP cells. (E) Western blot analysis for the expression of CXCR4 in A549 and A549/DDP cells. (F) Immunofluorescent assay for the expression of CXCR4 in A549 and A549/DDP cells. ${ }^{* * *} \mathrm{P}<0.001$.

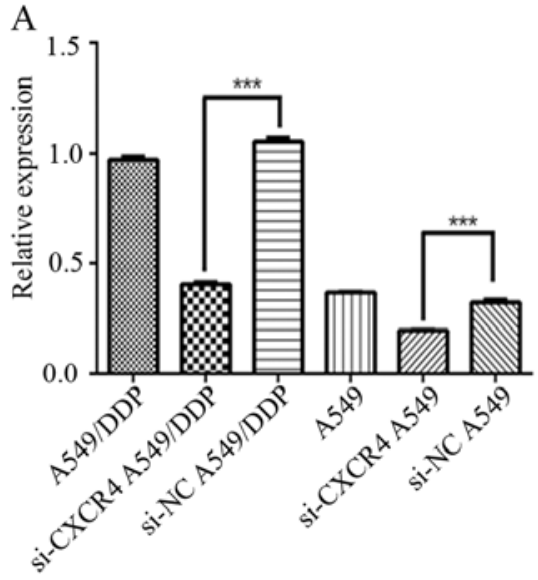

D

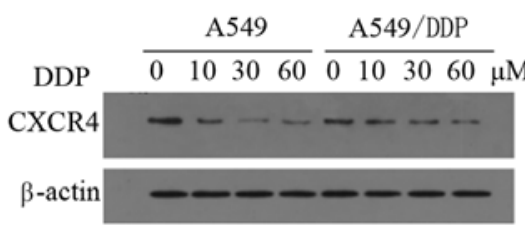

B

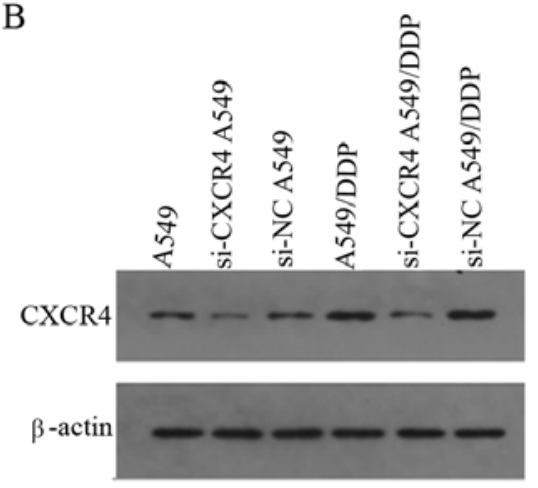

$\mathrm{E}$

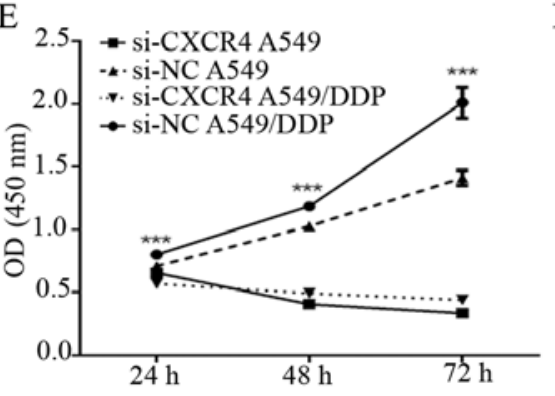

$\mathrm{C}$

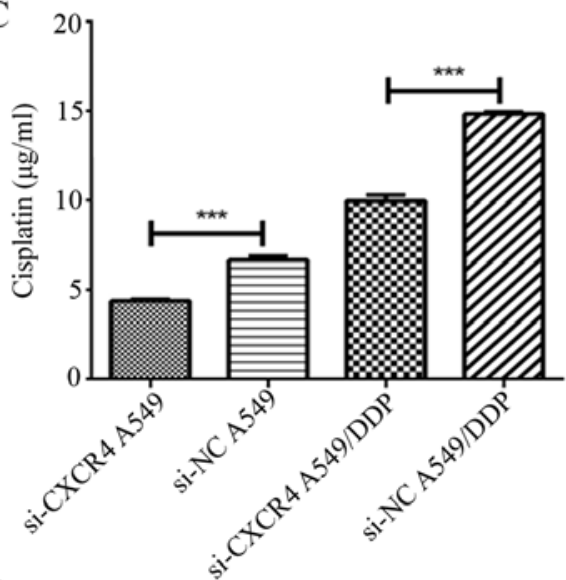

$\mathrm{F}$

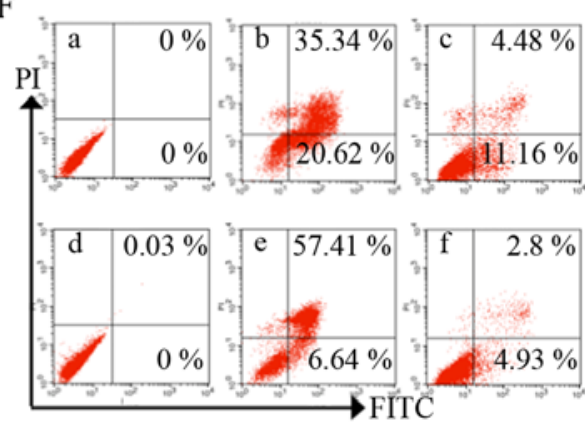

Figure 2. Effect of cisplatin in A549 and A549/DDP cells. (A and B) After CXCR4 siRNA transfection, CXCR4 mRNA was determined by qPCR (A) and protein expression was measured by western blot analysis (B). (C) Chemosensitivity assay was conducted at $48 \mathrm{~h}$ after cisplatin treatment. Values indicate mean $\mathrm{IC}_{50} \pm$ SEM. (D) CXCR4 protein was determined after treated with the different concentrations of cisplatin by western blot analysis. (E) Growth curves from siRNA transfected cells assessed by CCK8 assay. (F) Ratio of apoptotic cells was determined by flow cytometry. 
Table II. The correlation of CXCR4 and cytochrome p450 molecules.

\begin{tabular}{lcc}
\hline & $\begin{array}{c}\text { GSE219 }(\mathrm{n}=72) \\
\mathrm{r}, \mathrm{P} \text {-value }\end{array}$ & $\begin{array}{c}\text { GSE271 }(\mathrm{n}=263) \\
\mathrm{r}, \text { P-value }\end{array}$ \\
\hline CYP1A1 & $0.014,0.819$ & $0.027,0.667$ \\
CYP1A2 & $0.022,0.721$ & $-0.07,0.255$ \\
CYP1B1 & $0.616,0.000$ & $0.538,0.000$ \\
CYP2C8 & $0.058,0.337$ & $-0.114,0.064$ \\
CYP2C9 & $-0.134,0.027$ & $0.028,0.657$ \\
CYP2C18 & $-0.135,0.026$ & $-0.028,0.654$ \\
CYP2C19 & $-0.25,0.000$ & $-0.173,0.005$ \\
CYP2E1 & $-0.135,0.026$ & $-0.087,0.161$ \\
CYP3A4 & $0.063,0.3$ & $0.033,0.597$ \\
CYP3A5 & $-0.409,0.000$ & $-0.118,0.055$ \\
CYP3A7 & $-0.264,0.000$ & $0.025,0.689$ \\
CYP3A43 & $-0.287,0.000$ & $-0.204,0.001$ \\
\hline
\end{tabular}

CXCR4 expression levels may be influenced by patient age and sex, thereby having an effect on the tumor staging, cancer subtype and drug resistance characteristics.

CXCR4 knockdown enhances cisplatin-induced apoptosis and inhibits cell growth. To investigate whether CXCR4 mediates chemotherapy resistance in A549 and A549/DDP cells, we investigated the effect of CXCR4 siRNA on CXCR4 expression. CXCR4 siRNA was observed to inhibit the mRNA and protein expression levels of CXCR4 in A549 and A549/DDP cells $(\mathrm{P}<0.0001)$ (Fig. 2A and $\mathrm{B})$. We further investigated whether CXCR4 knockdown using CXCR4 siRNA had an effect on cisplatin sensitivity in NSCLC cells, of which it was observed that the half-maximal inhibitory concentration $\left(\mathrm{IC}_{50}\right)$ after $48 \mathrm{~h}$ was $6.665 \pm 0.215$ and $4.373 \pm 0.077 \mu \mathrm{g} / \mathrm{ml}$ for A549 and si-CXCR4 A549 cells, respectively $(\mathrm{P}<0.0001)$, while it was found to be $14.837 \pm 0.099$ and $9.969 \pm 0.318 \mu \mathrm{g} / \mathrm{ml}$ for A549/DDP and si-CXCR4 A549/DDP cells, respectively $(\mathrm{P}<0.0001)$. Both the A549 and A549/DDP si-CXCR4 transfected cells were more sensitive to cisplatin treatment than the non-transfected A549 and A549/DDP cells, indicating that CXCR4 knockdown increases sensitivity to cisplatin (Fig. 2C).

In addition, cisplatin effectively inhibited CXCR4 expression in both cells in a dose-dependent manner (Fig. 2D). CCK-8 assay was further applied to confirm the effect of CXCR4 knockdown on proliferation in A549 and A549/DDP cells. As shown in Fig. 2E, si-CXCR4 significantly suppressed cell proliferation in A549 and A549/DDP from 24 to $72 \mathrm{~h}$ $(\mathrm{P}<0.0001)$. Flow cytometry results indicated that A549 and A549/DDP transfected with negative control siRNA had an apoptotic cell percentage of $\sim 11.16$ and $4.93 \%$, respectively, while the percentage of apoptotic cells in the CXCR4 siRNA cells was 20.62 and $6.64 \%$, respectively (Fig. 2F). These results also suggest that knockdown of CXCR4 with siRNA-
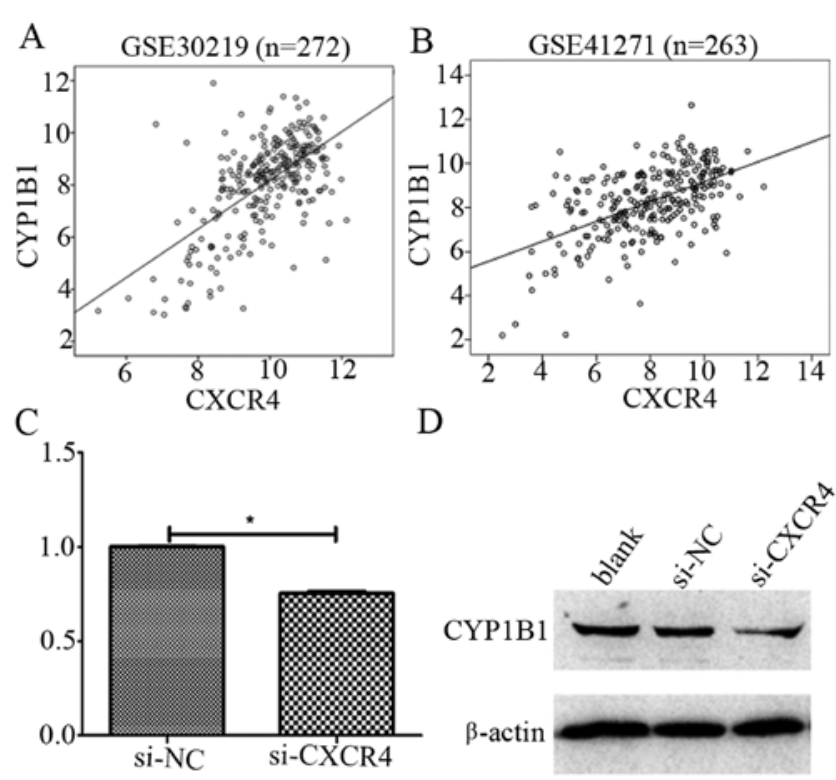

$\mathrm{D}$

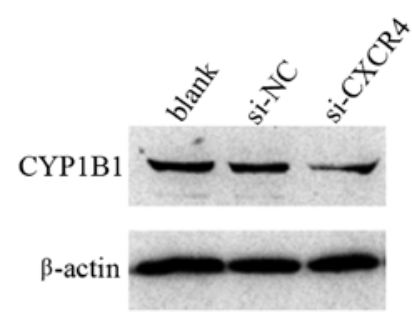

Figure 3. CXCR4 is upregulated with the expression of CYP1B1. (A) Expression of CYP1B1 associated with CXCR4 in GEO30219 dataset. (B) Expression of CYP1B1 associated with CXCR4 in GEO41271 dataset. (C) qPCR analysis of the expression of CYP1B1 after treatment with CXCR4 siRNA (50 nM, $48 \mathrm{~h}$ ). (D) Western blot analysis for the expression of CYP1B1 after treatment with CXCR4 siRNA (50 nM, $72 \mathrm{~h}$ ). GAPDH was detected as a loading control. All experiments were performed at least in triplicate. ${ }^{* * *} \mathrm{P}<0.001$.

contributes to the recovery of cisplatin-induced apoptosis in A549 and A549/DDP cells.

CXCR4 promotes cisplatin resistance through upregulation of CYPIB1. Increasing evidence has shown that many cytotoxic drugs are metabolized by cytochrome p450 (CYP) enzymes (17-25), we therefore explored the correlation of CXCR4 with CYP-related molecules (CYP1A1, CYP1A2, CYP1B1, CYP2C8, CYP2C9, CYP2C18, CYP2C19, CYP2E1, CYP3A4, CYP3A5, CYP3A7 and CYP3A43) by bioinformatics (Table II). Our results indicated a positive correlation between CXCR4 and CYP1B1 with $\mathrm{r}=0.616(\mathrm{P}<0.0001)$ and $\mathrm{r}=0.538$ $(\mathrm{P}<0.0001)$ in GSE30219 and GSE41271 datasets, respectively (Fig. 3A and B). Furthermore, we investigated whether CXCR4 plays an important role in cisplatin resistance in NSCLC by targeting CYP1B1, by examining the CYP1B1 mRNA and protein expression levels after CXCR4 knockdown by siRNA in A549/DDP cells. It was observed that CYP1B1 mRNA and protein level were significantly downregulated as a result of CXCR4 knockdown (Fig. 3C and D). Taken together, these results demonstrate CXCR4 may promote cisplatin resistance by regulating the expression levels of CYP1B1.

CYPIB1 regulates $N S C L C$ cell chemotherapy resistance. To evaluate the effects of CYP1B1 on NSCLC cell survival and resistance to chemotherapy, we examined whether reduction of CYP1B1 levels affects NSCLC cell survival. Transfection with CYP1B1 siRNA resulted in a marked reduction in endogenous levels of CYP1B1 in A549/DDP by western blot analysis (Fig. 4A). Knockdown of CYP1B1 significantly decreased the survival rate of A549/DDP cells, but did not decrease the survival rate of A549 cells (Fig. 4B). A549 and A549/DDP transfected with negative control siRNA were observed to have 
A

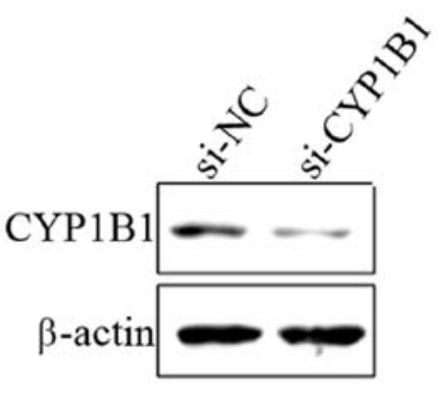

B

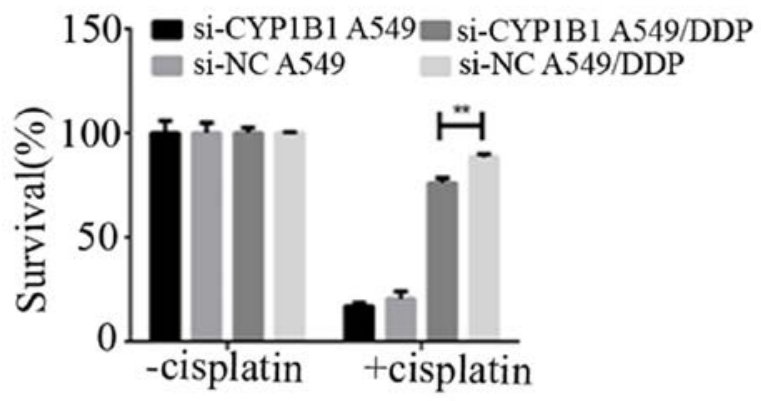

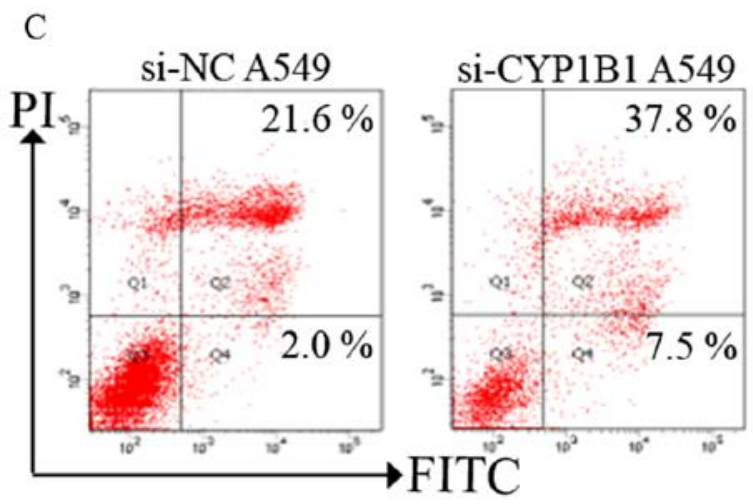
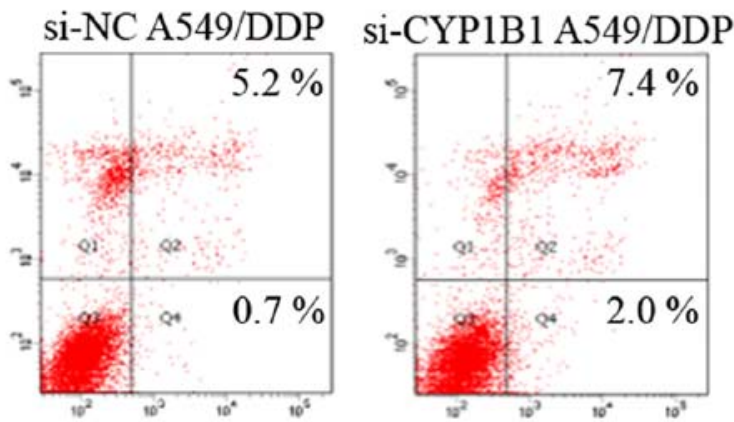

Figure 4. CYP1B1 knockdown sensitizes NSCLC cells to cisplatin in vitro. (A) Western blot analysis of the interference efficiency of CYP1B1 siRNA in A549 and A549/DDP. (B) CCK8 assay for the percentage of survival cells in A549 or A549/DDP cells when treated with/without si-CYP1B1. (C) Flow cytometry assay for the ratio of apoptotic cells in A549 or A549/DDP cells when treated with/without si-CYP1B1.

A
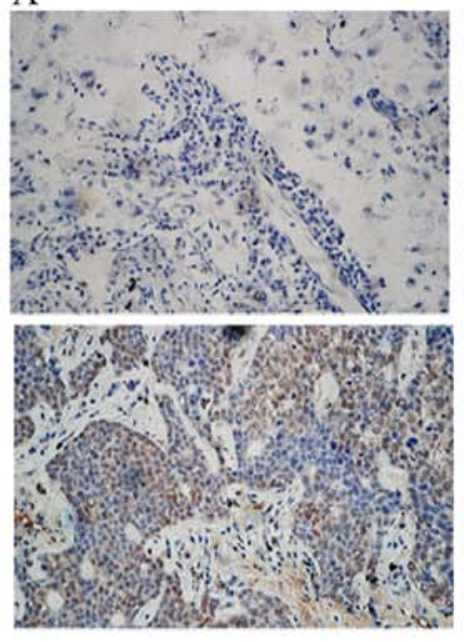
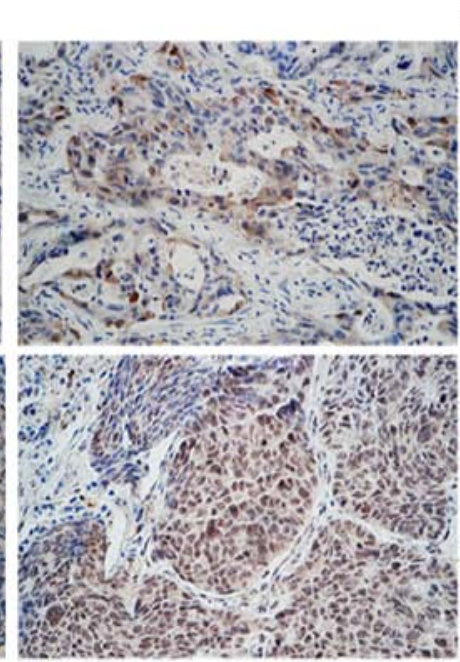

B

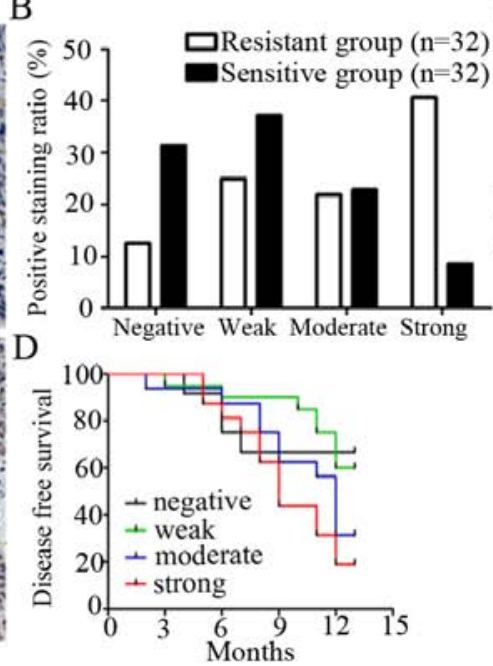

$\mathrm{C}$
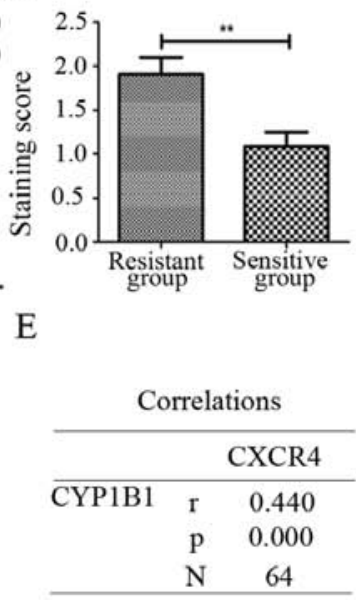

Figure 5. CYP1B1 overexpression was associated with poor survival in cisplatin-resistant NSCLC. (A) Representative images of NSCLC by immunohistochemistry (magnification, $\mathrm{x} 40$ ). (B) Positive staining ratio of cisplatin-resistant and sensitive group. (C) Immunohistochemistry staining score of cisplatin-resistant and sensitive group. (D) Kaplan-Meier estimate of disease-free survival for 64 patients with NSCLC according to the expression of CYP1B1. (E) Correlation of CXCR4 and CYP1B1 expression in 64 NSCLC by immunohistochemistry.

apoptotic cell percentages of 2.0 and $0.7 \%$, respectively, while the percentage of apoptotic cells was observed to increase to 7.5 and $2.0 \%$ in CYP1B1 knockdown cells, respectively (Fig. 4C).

Taken together, it was observed that the percentage of apoptosis was significantly increased in A549 and A549/ DDP cells after inhibiting CYP1B1 expression by siRNA, thus suggesting that CYP1B1 may play a significant role in NSCLC cell survival and resistance to chemotherapy.
CYPIB1 overexpression is associated with poor survival and cisplatin resistance in NSCLC. To further investigate the association between CYP1B1 and cisplatin resistance in NSCLC, we examined the expression of CYP1B1 in 32 cisplatin-sensitive NSCLC tissues and 32 cisplatin-resistant NSCLC tissues by immunohistochemistry. The expression levels of CYP1B1 in most cisplatin-sensitive tissues was observed to range from weak to undetectable, while that in cisplatin-resistant tissues ranged from weak to strong. Thus we categorized the NSCLC 
cisplatin-sensitive group into negative (25.0\%), weak (37.5\%), moderate (28.1\%) and strong (9.4\%), while the cisplatinresistant group was categorized into negative (12.6\%), weak (25.0\%), moderate (21.9\%) and strong (40.6\%) (Fig. 5A and B), the staining scores being $1.69 \pm 1.19$ in the cisplatin-resistant group and $1.08 \pm 0.97$ in cisplatin-sensitive group (Fig. 5C).

Having analyzed the CYP1B1 expression levels in cisplatin-sensitive and resistance groups, we further went on and analyzed CYP1B1 expression with associated patient clinical outcomes of all 64 NSCLC tissues. It was observed that expression of CYP1B1 correlated with disease-free survival and strong expression thus staining of CYP1B1 suggested poor survival as compared to moderate, weak and negative CYP1B1 expression and staining (Fig. 5D). Lastly, we confirmed that CXCR4 has a positive correlation with CYP1B1 ( $r=0.44$, $\mathrm{P}<0.001$ ) in NSCLC patients (Fig. 5E). These results suggest that CYP1B1 overexpression is associated with poor survival and cisplatin resistance in NSCLC.

\section{Discussion}

Cisplatin-based chemotherapy is one of the effective methods used in treatment of malignancies, however, cisplatin resistance has become a major therapeutic obstacle in clinical practice, and as such, much attention has been paid to the underlying mechanisms of chemotherapy resistance. In recent years, increasing evidence has revealed that CXCR4 is involved in chemoresistance in several types of cancers, including mantle cell lymphoma (26), chronic myelogenous leukemia (27) and breast cancer (28). However, the function of CXCR4 in NSCLC chemoresistance remains unknown. In this study, we explored the potential role of CXCR4 in NSCLC chemotherapeutic responses.

We investigated the possible association of CXCR4 high expression with cancer progression and poor prognosis in NSCLC tissues. Thus, we examined the expression of CXCR4 in 64 NSCLC patient samples. We observed that patient samples with a high CXCR4 expression significantly associated with a shorter disease-free patient survival as seen in the cisplatin-resistant group, when compared with the cisplatin-sensitive group, thereby suggesting that CXCR4 high expression might be closely related with chemoresistance. This was further amplified when we analyzed the clinicopathological characteristics in relation to the CXCR4 expression, in which we observed that CXCR4 was mostly expressed in male patients $>65$ years old and associated with the tumor size and drug resistance levels. Other researchers have also reported the possible association of CXCR4 expression levels with patient sex and tumor staging $(29,30)$.

Some studies have previously revealed the association between CXCR4 overexpression and chemoresistance in some cancers. Nakamura et al observed that CXCR4 overexpression promoted drug resistance in cancer cells by upregulating transforming growth factor- $\beta 2$ (12). Similarly, Sison et al reported that CXCR4 overexpression protected pediatric acute lymphoblastic leukemia cells from chemotherapy-induced apoptosis, thus enhancing chemoresistance when the cells were co-cultured with bone marrow stroma. Treatment with a CXCR4 inhibitor plerixafor diminished the protective effect of apoptosis, thus conferring chemosensitivity (31). High CXCR4 expression levels have also been significantly associated with cisplatin-based chemotherapeutic resistance in epithelial ovarian cancer (13).

In this study, by using cisplatin-resistant NSCLC cells A549/DDP and its parental A549 cells, we demonstrated that CXCR4 might play an important role in regulating the chemoresistance in NSCLC, as it was observed that CXCR4 was highly expressed in A549/DDP cells and CXCR4 knockdown resulted in reduced proliferation and cell apoptosis induction. Analyses of the $\mathrm{IC}_{50}$ values also indicated that CXCR4 knockdown resulted in increased chemosensitivity of A549/DDP cells to cisplatin.

Therefore, in order to define the mechanisms of CXCR4 associated NSCLC chemoresistance, we further downloaded two largest datasets of NSCLC patients from gene expression omnibus and performed a correlation analysis with CYP related-molecules. We found only CYP1B1 had a positive correlation with CXCR4. CYP1B1 belongs to cytochrome p450 family, which is a multi-gene family of enzymes implicated in the metabolism of a diverse range of xenobiotics and endogenous compounds (24). Several researchers have proposed interesting concepts, in explaining the mechanisms of CYP1B1 induced cytotoxic effect reduction of chemotherapeutic drug $(20,21,23,25)$, as other have mainly focused on CYP1B1 polymorphism in cancer process $(22,32,33)$. Of particular interest, Patel et al reported that CYP1B1 may be regulated by cytokines, as interleukin- 6 was observed to mediate of CYP1B1 upregulation via DNA methylation in colorectal cancer (34). Therefore, we further investigated the differential expression of CYP1B1 between the chemosensitive and chemoresistance groups, and we observed that CYP1B1 levels were significantly increased in the chemoresistance group. We also observed that CYP1B1 knockdown decreased the cell survival rates, enhanced cell apoptosis and partially reversed the chemoresistance of cisplatin in cancer cells. Additionally, we also observed that CXCR4 mediated cisplatin resistance via regulating CYP1B1 expression.

In conclusion, this study shows that CXCR4 mediates cisplatin chemoresistance in NSCLC in a CYP1B1-dependent manner. Thus, it could be used as a potential therapeutic target in NSCLC chemoresistance patients and as a clinically useful predictor of cisplatin response.

\section{Acknowledgements}

This study was supported by National Natural Science Foundation of China (nos. 81270607 and 81541027) and Natural Science Foundation of Hubei (nos. 2014CFA070 and 2015CFB653).

\section{References}

1. Torre LA, Bray F, Siegel RL, Ferlay J, Lortet-Tieulent J and Jemal A: Global cancer statistics, 2012. CA Cancer J Clin 65: 87-108, 2015.

2. Siegel R, Naishadham D and Jemal A: Cancer statistics, 2013. CA Cancer J Clin 63: 11-30, 2013.

3. DeSantis CE, Lin CC, Mariotto AB, Siegel RL, Stein KD, Kramer JL, Alteri R, Robbins AS and Jemal A: Cancer treatment and survivorship statistics, 2014. CA Cancer J Clin 64: 252-271, 2014. 
4. Holohan C, Van Schaeybroeck S, Longley DB and Johnston PG: Cancer drug resistance: An evolving paradigm. Nat Rev Cancer 13: 714-726, 2013.

5. Teicher BA and Fricker SP: CXCL12 (SDF-1)/CXCR4 pathway in cancer. Clin Cancer Res 16: 2927-2931, 2010.

6. Dai X, Mao Z, Huang J, Xie S and Zhang H: The CXCL12/ CXCR4 autocrine loop increases the metastatic potential of non-small cell lung cancer in vitro. Oncol Lett 5: 277-282, 2013.

7. Xie S, Zeng W, Fan G, Huang J, Kang G, Geng Q, Cheng B, Wang W and Dong P: Effect of CXCL12/CXCR4 on increasing the metastatic potential of non-small cell lung cancer in vitro is inhibited through the downregulation of CXCR4 chemokine receptor expression. Oncol Lett 7: 941-947, 2014.

8. Wang T, Mi Y, Pian L, Gao P, Xu H, Zheng Y and Xuan X: RNA targeting CXCR4 inhibits proliferation and invasion of esophageal carcinoma cells. Diagn Pathol 8: 104, 2013.

9. Nikzaban M, Hakhamaneshi MS, Fakhari S, Sheikhesmaili F, Roshani D, Ahsan B, Kamali F and Jalili A: The chemokine receptor CXCR4 is associated with the staging of gastric cancer. Adv Biomed Res 3: 16, 2014.

10. Teng F, Tian WY, Wang YM, Zhang YF, Guo F, Zhao J, Gao C and Xue FX: Cancer-associated fibroblasts promote the progression of endometrial cancer via the SDF-1/CXCR4 axis. J Hematol Oncol 9: 8, 2016.

11. Okuyama Kishima M, de Oliveira CE, Banin-Hirata BK, Losi-Guembarovski R, Brajão de Oliveira K, Amarante MK and Watanabe MA: Immunohistochemical expression of CXCR4 on breast cancer and its clinical significance. Anal Cell Pathol (Amst) 2015: 891020, 2015.

12. Nakamura T, Shinriki S, Jono H, Guo J, Ueda M, Hayashi M, Yamashita S, Zijlstra A, Nakayama H, Hiraki A, et al: Intrinsic TGF- 32 -triggered SDF-1-CXCR4 signaling axis is crucial for drug resistance and a slow-cycling state in bone marrowdisseminated tumor cells. Oncotarget 6: 1008-1019, 2015

13. Li J, Jiang K, Qiu X, Li M, Hao Q, Wei L, Zhang W, Chen B and Xin X: Overexpression of CXCR4 is significantly associated with cisplatin-based chemotherapy resistance and can be a prognostic factor in epithelial ovarian cancer. BMB Rep 47: 33-38, 2014

14. Rousseaux S, Debernardi A, Jacquiau B, Vitte AL, Vesin A, Nagy-Mignotte H, Moro-Sibilot D, Brichon PY, Lantuejoul S, Hainaut $\mathrm{P}$, et al: Ectopic activation of germline and placental genes identifies aggressive metastasis-prone lung cancers. Sci Transl Med 5: 186ra66, 2013

15. Sato M, Larsen JE, Lee W, Sun H, Shames DS, Dalvi MP, Ramirez RD, Tang H, DiMaio JM, Gao B, et al: Human lung epithelial cells progressed to malignancy through specific oncogenic manipulations. Mol Cancer Res 11: 638-650, 2013.

16. Riquelme E, Suraokar M, Behrens C, Lin HY, Girard L, Nilsson MB, Simon G, Wang J, Coombes KR, Lee JJ, et al: VEGF/VEGFR-2 upregulates EZH2 expression in lung adenocarcinoma cells and EZH2 depletion enhances the response to platinum-based and VEGFR-2-targeted therapy. Clin Cancer Res 20: 3849-3861, 2014

17. Chang I, Mitsui Y, Fukuhara S, Gill A, Wong DK, Yamamura S, Shahryari V, Tabatabai ZL, Dahiya R, Shin DM, et al: Loss of miR-200c up-regulates CYP1B1 and confers docetaxel resistance in renal cell carcinoma. Oncotarget 6: 7774-7787, 2015.

18. Hendrikx JJ, Lagas JS, Rosing H, Schellens JH, Beijnen JH and Schinkel AH: P-glycoprotein and cytochrome P450 3A act together in restricting the oral bioavailability of paclitaxel. Int $\mathbf{J}$ Cancer 132: 2439-2447, 2013.

19. Kudo T, Ozaki Y, Kusano T, Hotta E, Oya Y, Komatsu S, Goda H and Ito K: Effect of buffer conditions on CYP2C8-mediated paclitaxel 6alpha-hydroxylation and CYP3A4-mediated triazolam alpha- and 4-hydroxylation by human liver microsomes. Xenobiotica 46: 241-246, 2016.
20. Laroche-Clary A, Le Morvan V, Yamori T and Robert J: Cytochrome P450 1B1 gene polymorphisms as predictors of anticancer drug activity: Studies with in vitro models. Mol Cancer Ther 9: 3315-3321, 2010

21. Pastina I, Giovannetti E, Chioni A, Sissung TM, Crea F, Orlandini C, Price DK, Cianci C, Figg WD, Ricci S, et al: Cytochrome 450 1B1 (CYP1B1) polymorphisms associated with response to docetaxel in castration-resistant prostate cancer (CRPC) patients. BMC Cancer 10: 511, 2010.

22. Vasile E, Tibaldi C, Leon GL, D'Incecco A and Giovannetti E: Cytochrome P450 1B1 (CYP1B1) polymorphisms are associated with clinical outcome of docetaxel in non-small cell lung cancer (NSCLC) patients. J Cancer Res Clin Oncol 141: 1189-1194, 2015.

23. Wen CJ, Wu LX, Fu LJ, Shen DY, Zhang X, Zhang YW, Yu J and Zhou HH: Preferential induction of CYP1A1 over CYP1B1 in human breast cancer MCF-7 cells after exposure to berberine. Asian Pac J Cancer Prev 15: 495-499, 2014.

24. Xu X, Zhang XA and Wang DW: The roles of CYP450 epoxygenases and metabolites, epoxyeicosatrienoic acids, in cardiovascular and malignant diseases. Adv Drug Deliv Rev 63: 597-609, 2011.

25. Zhu Z, Mu Y, Qi C, Wang J, Xi G, Guo J, Mi R and Zhao F: CYP1B1 enhances the resistance of epithelial ovarian cancer cells to paclitaxel in vivo and in vitro. Int J Mol Med 35: 340-348, 2015.

26. Chen Z, Teo AE and McCarty N: ROS-induced CXCR4 signaling regulates mantle cell lymphoma (MCL) cell survival and drug resistance in the bone marrow microenvironment via autophagy. Clin Cancer Res 22: 187-199, 2016.

27. Wang Y, Miao H, Li W, Yao J, Sun Y, Li Z, Zhao L and Guo Q: CXCL12/CXCR4 axis confers adriamycin resistance to human chronic myelogenous leukemia and oroxylin A improves the sensitivity of K562/ADM cells. Biochem Pharmacol 90: 212-225, 2014.

28. De Luca A, D'Alessio A, Gallo M, Maiello MR, Bode AM and Normanno N: Src and CXCR4 are involved in the invasiveness of breast cancer cells with acquired resistance to lapatinib. Cell Cycle 13: 148-156, 2014.

29. Wang M, Chen GY, Song HT, Hong X, Yang ZY and Sui GJ: Significance of CXCR4, phosphorylated STAT3 and VEGF-A expression in resected non-small cell lung cancer. Exp Ther Med 2: 517-522, 2011

30. Al Zobair AA, Al Obeidy BF, Yang L, Yang C, Hui Y, Yu H, Zheng F, Yang G, Xie C, Zhou F, et al: Concomitant overexpression of EGFR and CXCR4 is associated with worse prognosis in a new molecular subtype of non-small cell lung cancer. Oncol Rep 29: 1524-1532, 2013

31. Sison EA, Magoon D, Li L, Annesley CE, Rau RE, Small D and Brown P: Plerixafor as a chemosensitizing agent in pediatric acute lymphoblastic leukemia: Efficacy and potential mechanisms of resistance to CXCR4 inhibition. Oncotarget 5: 8947-8958, 2014

32. Sankhwar M, Sankhwar SN, Abhishek A, Gupta N and Rajender S: CYP1B1 gene polymorphisms correlate with an increased risk of urinary bladder cancer in India. Urol Oncol 34: 167 e161-168, 2016.

33. Yu PJ, Chen WG, Feng QL, Chen W, Jiang MJ and Li ZQ: Association between CYP1B1 gene polymorphisms and risk factors and susceptibility to laryngeal cancer. Med Sci Monit 21: 239-245, 2015.

34. Patel SA, Bhambra U, Charalambous MP, David RM, Edwards RJ, Lightfoot T, Boobis AR and Gooderham NJ: Interleukin-6 mediated upregulation of CYP1B1 and CYP2E1 in colorectal cancer involves DNA methylation, miR27b and STAT3. Br J Cancer 111: 2287-2296, 2014. 